\title{
Predictive Value of HbA1c-levels with Regard to In-hospital Mortality, Length of Hospital Stay and Intensive Care Utilisation versus Different Emergency Risk Scores and the Manchester Triage System in Unselected Medical Emergency Admissions
}

\author{
Markus Sander $1, \star$, Johannes Fickler ${ }^{2}$, Uwe Neddermeyer ${ }^{3}$, \\ Stefan Von Delius ${ }^{4}$, Stephan Budweiser ${ }^{5}$
}

\author{
${ }^{1}$ Private Practice, \\ Hildegard-von-Bingen-Str. 1, 93053 \\ Regensburg, Germany \\ ${ }^{2}$ Private Practice, Rupertistraße $11 \mathrm{~A}$, \\ 84518 Garching an der Alz, Germany \\ ${ }^{3}$ Department of Emergency Medicine, \\ RoMed Clinical Centre Rosenheim, \\ Rosenheim, Germany \\ ${ }^{4}$ Department of Internal Medicine II, \\ RoMed Clinical Centre Rosenheim, \\ Rosenheim, Germany \\ ${ }^{5}$ Department of Internal Medicine III, \\ Division of Pulmonary and Respiratory \\ Medicine, RoMed Clinical Centre \\ Rosenheim, Rosenheim, Germany \\ *Correspondence \\ markus_sander@gmx.net \\ (Markus Sander)
}

\begin{abstract}
Objectives: To evaluate the predictive value of HbAlc levels in medical patients admitted to the emergency department (ED) regarding in-hospitalmortality, length of stay (LOS) and transferral to intensive care unit (ICU) and to compare them with different physiologically based emergency scoring systems and the Manchester Triage System (MTS). Methods: In a prospective cohortstudy, 1117 consecutive patients presenting to the medical ED were assessed. Data collected included age, sex, vital signs, temperature, oxygen saturation, respiratory rate, AVPU (Alert; Verbal response; response to Pain; Unresponsive)score, MTS, different emergency scores and HbA1c. The data were correlated with LOS, hospital mortality and intensive care utilisation. Results: HbA1c had similar accuracy in predicting LOS as most physiologically based scores (AUC = $0.568, \mathrm{p}=0.688$ to 0.714$)$ and ICU utilisation $(\mathrm{AUC}=0.525, \mathrm{p}=0.001$ compared with MTS, for all others $\mathrm{p}=0.077$ to 0.830 ). HbA1c was positively correlated with LOS and ICU-transferral but correlated poorly with mortality, resulting in low predictive power (AUC $=0.501, \mathrm{p}=0.033$ to 0.845 ). The subgroups with HbA1c below the median and below $6.5 \%$ had a shorter $\operatorname{LOS}(\mathrm{p}=0.012$ and $\mathrm{p}=$ 0.004). The differences for other subgroups were not significant. Conclusions: HbA1c was positively correlated with LOS and ICU-referral, reflecting higher health-care utilisation, indicating that it may be a useful parameter in evaluating severity of illness in emergency patients.
\end{abstract}

\section{Keywords}

Glycated haemoglobin, Emergency score, Manchester Triage System, Mortality, Length of stay, ICU referral

\section{Introduction}

In order to provide assistance in efficiently allocating resources in the emergency department (ED), a large variety of risk assessment systems have been proposed for triaging patients [1,9]. One of the most widely used protocols is the Manchester Triage System (MTS) which is based on major symptoms/complaints. Additionally, several scoring systems based on measurable physiological values have been developed. The most common systems are variations of the Early Warning Score System. These scores differ in the composition and weighting of the measured vital signs and other parameters. Most incorporate a combination of respiratory rate, heart rate, temperature, blood pressure, oxygen saturation and the AVPU(Alert; Verbal response; response to Pain; Unresponsive)-score. Some exclude one or more of these variables, others add additional variables like urine output, age, sex, respiratory support, a pain 
TA B L E 1. Scoring systems.

CART

\begin{tabular}{|lcc|}
\hline Vital sign & value & score \\
\hline RR & $<21$ & 0 \\
& $21-23$ & 8 \\
& $24-25$ & 12 \\
& $26-29$ & 15 \\
\hline$>29$ & 22 & \\
\hline HR & $<110$ & 0 \\
& $110-139$ & \\
& $>139$ & 13 \\
\hline DBP & $>49$ & 0 \\
\hline & $40-49$ & 4 \\
& $35-39$ & 6 \\
\hline & $<35$ & 13 \\
\hline Age & $<55$ & 0 \\
\hline & $55-69$ & 4 \\
\hline & $>69$ & 9 \\
\hline
\end{tabular}

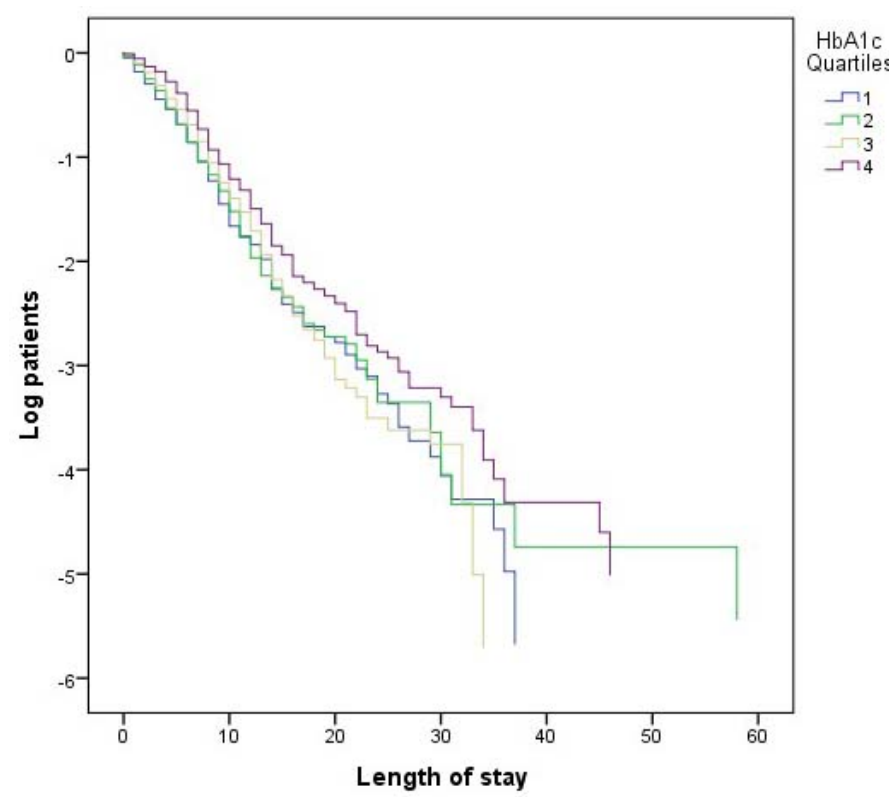

FIGURE 1. Logarithmic Kaplan Maier curve of length of stay. $1: H b A 1 c \leq 5.4 \% ; 2: H b A l c>5.4 \%$ to $<$ 5.8\%; 3: HbAlc 5.8\% to $<6.3 \%$; 4: HbAlc $\geq 6.3 \%$, *: $p=$ 0.010 . The mean length of stay was significantly prolonged in the fourth quartile.

scale or substitute the Glasgow Coma Scale for AVPU. However, there is uncertainty regarding the most adequate tool for prediction of severity of illness and the demand for available resources [1].

As one of the primary problems is the correct implementation of well-established systems, ease of use is of great importance. An ideal parameter should be easily and quickly obtainable.

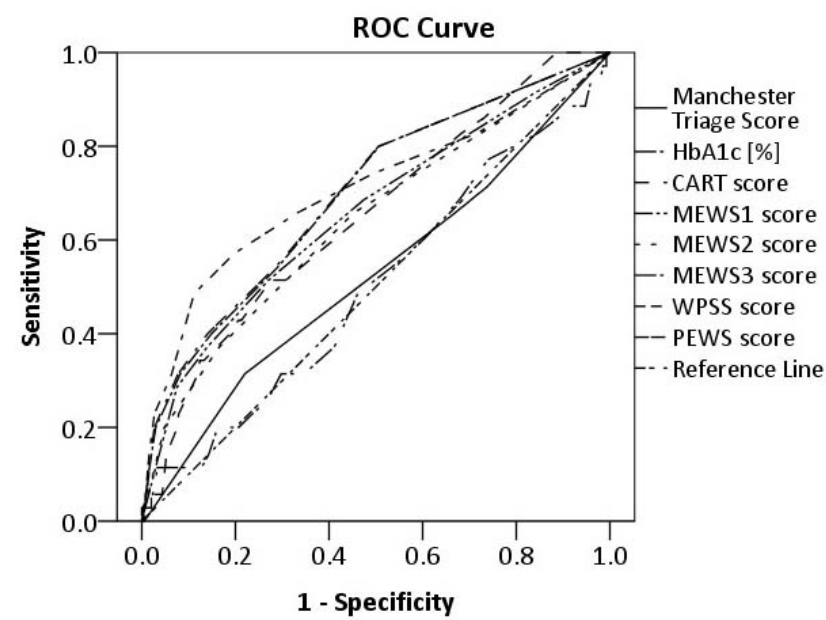

F IGURE 2. Prediction of mortality. ROC curves for the examined systems regarding mortality.Graphic depiction of AUCs for the prediction of mortality.

Cardiovascular events and metabolic derangements are the most common reasons for rapid, unexpected clinical deterioration and unfavorable course of the disease. Specifically, glycated hemoglobin $(\mathrm{HbAlc})$ has been shown to be linked to adverse cardiovascular outcomes [10], adverse outcomes for sepsis in diabetic patients [11], increased mortality in cerebrovascular disease [12] and increased allcause mortality [13]. Point-of-care assays that allow quick and reliable $\mathrm{HbA} 1 \mathrm{c}$ measurements have been developed recently and are increasingly utilised in EDs [14]. Considering the high numbers of undetected cases of diabetes mellitus [15] and its rapidly increasing incidence [16], we hypothesized that $\mathrm{HbAlc}$ could be useful for estimation of clinical outcomes in unselected emergency room patients. Accordingly, we compared the predictive value of $\mathrm{HbAlc}$ to established measures for triage in the ED.

\section{Methods}

This study was approved by the ethical review committee of the University of Regensburg (No 14-101-0008). The research is in accordance with the Helsinki Declaration of 1975, as revised in 2010.

Data collection was in accordance with Bavarian law (BayKrG, Art. 27).

\subsection{Study population}

Within a prospective observational design, we enrolled consecutive patients who presented to the ED of the RoMed Hospital of Rosenheim, Germany, between June 5, 2014 and August 15, 2014. RoMed Klinikum Rosenheim is a major regional secondary care hospital in southern Bavaria with 640 beds, where approximately 27,000 inpatients and 35,000 outpatients are treated annually. 
TA B L E 1. Continued.

MEWS1

\begin{tabular}{|c|c|c|c|c|c|c|c|}
\hline Score & 3 & 2 & 1 & 0 & 1 & 2 & 3 \\
\hline RR & & $\leq 8$ & & $9-14$ & $15-20$ & $21-29$ & $>29$ \\
\hline HR & & $\leq 40$ & $41-50$ & $51-100$ & $101-110$ & $111-129$ & $>129$ \\
\hline SBP & $\leq 70$ & $71-80$ & $81-100$ & 101-199 & & $\geq 200$ & \\
\hline Temp & & $\leq 35$ & $35.1-36$ & $36.1-38$ & $38.1-38.5$ & $\geq 38.6$ & \\
\hline AVPU & & & & A & V & $\mathrm{P}$ & $\mathrm{U}$ \\
\hline \multicolumn{8}{|c|}{ MEWS2 } \\
\hline Score & 3 & 2 & 1 & $\mathbf{0}$ & 1 & 2 & 3 \\
\hline $\mathrm{RR}$ & & & & $<24$ & $\geq 24$ & & \\
\hline HR & & $\leq 40$ & & $40-129$ & $\geq 130$ & & \\
\hline SBP & & $<80$ & $80-85$ & $>85$ & & & \\
\hline Age & & & & $<80$ & $80-89$ & $\geq 90$ & \\
\hline AVPU & & & & $\mathrm{A}$ & $\mathrm{V}$ & $\mathrm{P} / \mathrm{U}$ & \\
\hline $\mathrm{SO} 2$ & & $<85$ & $85-89$ & $\geq 90$ & & & \\
\hline \multicolumn{8}{|c|}{ MEWS3 } \\
\hline Score & 3 & 2 & 1 & $\mathbf{0}$ & 1 & 2 & 3 \\
\hline $\mathrm{RR}$ & & $<9$ & & $9-14$ & $15-20$ & $21-29$ & $\geq 30$ \\
\hline HR & & $\leq 40$ & $41-50$ & $51-100$ & $101-110$ & $111-129$ & $\geq 130$ \\
\hline SBP & $\leq 70$ & $71-80$ & $81-100$ & 100-199 & & $\geq 200$ & \\
\hline Temp & $<35$ & & & $35.0-38.4$ & & $\geq 38.5$ & \\
\hline AVPU & & & & A & V & $\mathrm{P}$ & $\mathrm{U}$ \\
\hline \multicolumn{8}{|l|}{ WPSS } \\
\hline Score & 3 & 2 & 1 & 0 & 1 & 2 & 3 \\
\hline $\mathrm{RR}$ & & & & $\leq 19$ & $20-21$ & $\geq 22$ & \\
\hline HR & & & & $\leq 101$ & $\geq 102$ & & \\
\hline SBP & & $\leq 99$ & & $\geq 100$ & & & \\
\hline Temp & $<35.3$ & & & $\geq 35.3$ & & & \\
\hline AVPU & & & & A & & & $\mathrm{V} / \mathrm{P} / \mathrm{U}$ \\
\hline $\mathrm{SO} 2$ & $<92$ & $92-93$ & 94-95 & $96-100$ & & & \\
\hline
\end{tabular}

PEWS

$\begin{array}{lccccccc}\text { Score } & \mathbf{3} & \mathbf{2} & \mathbf{1} & \mathbf{0} & \mathbf{1} & \mathbf{2} & \mathbf{3} \\ \text { RR } & & \leq 8 & & 9-14 & 15-20 & 21-29 & >30 \\ \text { HR } & & \leq 40 & 41-50 & 51-100 & 101-110 & 111-130 & >130 \\ \text { SBP } & \leq 70 & 71-80 & 81-100 & 100-199 & & >200 & \\ \text { Temp } & & \leq 35.0 & 35.1-36.0 & 36.1-37.9 & 38.0-38.9 & \geq 39 & \\ \text { AVPU } & & & & \mathrm{A} & \mathrm{V} & \mathrm{P} & \mathrm{U}\end{array}$

Examined scoring systems and their composition Abbreviations and units: Age [years]; AVPU [Alert, Voice, Pain, Unresponsive]; CART, Cardiac Arrest Risk Triage; DBP, diastolic blood pressure [mmHg]; HR, heart rate [beats/min]; MEWS, Modified Early Warning System; PEWS, Patientrack Early Warning System; RR, respiratory rate [breaths/min]; $\mathrm{SBP}$, systolic blood pressure [mmHg]; SO2, oxygen saturation [\%];Temp, temperature $\left[{ }^{\circ} \mathrm{C}\right] ;$ WPSS, Worthington Physiological Scoring System; CART, MEW, PEW (please add the meaning and order the abbreviations alphabetically).

\subsection{Data collection}

In addition to the standard operating procedure in the ED, the following data were collected upon presentation: age, sex, heart rate (HR), non-invasive systolic and diastolic blood pressure, respiratory frequency (as measured 
TA B L E 2. Patient characteristics.

\begin{tabular}{lc} 
Age, median (IQR), y & $73(61-81)$ \\
Male sex, No. (\%) & $606(54.3 \%)$ \\
\hline Heartrate, median (IQR), /min & $85(71-100)$ \\
Respiratory rate, median (IQR), /min & $18(15-20)$ \\
Systolic blood pessure, median (IQR), mmHg & $138(120-157)$ \\
\hline Diastolic blood pressure, median (IQR), mmHg & $76(65-88)$ \\
Temperature, median (IQR), ${ }^{\circ} \mathrm{C}$ & $36.8(36.5-37.3)$ \\
\hline Oxygen saturation, median (IQR), \% & $96(94-97)$ \\
\hline HbAlc, median (IQR), mmol & $39.9(35.5-45.4)$ \\
\hline HbA1c, median (IQR), \% & $5.8(5.4-6.3)$ \\
\hline AVPU: & \\
Alert, No., \% & $1004(89.9 \%)$ \\
\hline Voice, No., \% & $105(9.4 \%)$ \\
Pain, No., \% & $0(0 \%)$ \\
Unresponsive, No., \% & $8(0.7 \%)$ \\
Manchester Triage System: & \\
Blue, No., \% & $6(0.5 \%)$ \\
Green, No., \% & $243(21.8 \%)$ \\
\hline Yellow, No., \% & $497(44.5 \%)$ \\
Orange, No., \% & $78(7.0 \%)$ \\
Red, No., \% & $293(26.2 \%)$ \\
\hline
\end{tabular}

Table 2: Characteristics of the tested population.

Abbreviations: IQR: Interquartile range; y: years.

via monitor), pulse oxygen saturation $\left(\mathrm{SaO}_{2}\right)$, body temperature, AVPU-Score [2]. The nurse in charge applied the standard examination within 20 minutes following presentation. Patients missing one or more data points were excluded.

Upon presentation at the ED, patients were stratified using the MTS [3] by specifically trained nurses according to protocol. MTS color levels were converted to an ordinal scale of 1 to 5 for statistical analysis.

Patients with complete data sets were followed until death, discharge from hospital or referral to another hospital. Additionally, we assessed length of hospital stay (LOS) and admission to the intensive care unit or intermediate care unit (summarized as ICU) at any timepoint during hospital stay.

HbAlc levels were determined by high pressure liquid chromatography (Tosoh Bioscience Inc, Japan) using EDTA venous blood samples acquired upon presentation.

The estimated catchment area of our hospital includes around 200000 people. The data set was to be used in several studies. To answer questions with a confidence level of $95 \%$ and a confidence interval of 3, a sample size of 1061 patients was calculated.

Potential biases included the misapplication of the MTS protocol which we tried to address by specifically instructing the nurses.

\subsection{Emergency scores}

The following scores were calculated (Table 1):

Cardiac Arrest Risk Triage (CART) [4], three different versions of the Modified Early Warning Score (MEWS1-3) [5-7], Worthington Physiological Scoring System (WPSS) [8], Central Manchester University Hospitals National Health Service Foundation Trust Early Warning Score, itself a variation of the EWS, used in the Patientrack Early Warning System (PEWS) [9].

The CART-Score was initially developed to specifically asses the risk of cardiac arrest but was included as an interesting alternative as it incorporates similar and easily available parameters that have been compared to general emergency scores before [4].

\subsection{Statistics}

Statistical analysis was performed using SPSS Statistics Version 24 (IBM Corp. Released 2016, IBM SPSS for Windows, Version 24.0. Armonk, NY: IBM Corp), significancy in AUC differences was determined using R Version 3.3.2 (R: A Language and Environment for Statistical Computing. R Foundation for Statistical Computing. Vienna, Austria. URL: www.R-project.org GNUlicense) and the R package pROC [17].

For continous parameters mean, standard deviation, median and range were calculated. For binary and categorial 
TA B L E 2 b. Areas under the curve for the prediction of mortality Area Under the Curve. Test Result Variable(s) Area Std. Error Asymptotic Sig. Asymptotic 95\% Confidence Interval

$\begin{array}{lccccc} & & & & \text { Lower Bound } & \text { Upper Bound } \\ \text { MTS } & 0.524 & 0.053 & 0.632 & 0.419 & 0.628 \\ \text { HbA1c } & 0.501 & 0.051 & 0.99 & 0.4 & 0.601 \\ \text { CART } & 0.651 & 0.047 & 0.002 & 0.558 & 0.744 \\ \text { MEWS1 } & 0.696 & 0.047 & <0.001 & 0.604 & 0.788 \\ \text { MEWS2 } & 0.64 & 0.052 & 0.005 & 0.539 & 0.741 \\ \text { MEWS3 } & 0.658 & 0.051 & 0.001 & 0.558 & 0.758 \\ \text { WPSS } & 0.707 & 0.053 & <0.001 & 0.603 & 0.812 \\ \text { PEWS } & 0.699 & 0.047 & <0.001 & 0.606 & 0.791\end{array}$

2b: Numeric values of AUCs as shown in Fig. $2 a$.

TA B L E 2 c. Comparison of AUCs and corresponding p-values.

\begin{tabular}{|c|c|c|c|c|}
\hline Predictor 1 & AUC1 & Predictor 2 & AUC2 & P-value \\
\hline MTS & 52.38 & HbAlc & 50.06 & 0.845 \\
\hline MTS & 52.38 & CART & 65.08 & 0.131 \\
\hline MTS & 52.38 & MEWS1 & 69.6 & 0.07 \\
\hline MTS & 52.38 & MEWS2 & 64 & 0.191 \\
\hline MTS & 52.38 & MEWS3 & 65.79 & 0.179 \\
\hline MTS & 52.38 & WPSS & 70.73 & 0.07 \\
\hline MTS & 52.38 & PEWS & 69.88 & 0.07 \\
\hline HbAlc & 50.06 & CART & 65.08 & 0.07 \\
\hline HbAlc & 50.06 & MEWS1 & 69.6 & 0.033 \\
\hline $\mathrm{HbAlc}$ & 50.06 & MEWS2 & 64 & 0.131 \\
\hline $\mathrm{HbA1c}$ & 50.06 & MEWS3 & 65.79 & 0.069 \\
\hline $\mathrm{HbA1c}$ & 50.06 & WPSS & 70.73 & 0.038 \\
\hline HbAlc & 50.06 & PEWS & 69.88 & 0.033 \\
\hline CART & 65.08 & MEWS1 & 69.6 & 0.434 \\
\hline CART & 65.08 & MEWS2 & 64 & 0.86 \\
\hline CART & 65.08 & MEWS3 & 65.79 & 0.876 \\
\hline CART & 65.08 & WPSS & 70.73 & 0.302 \\
\hline CART & 65.08 & PEWS & 69.88 & 0.416 \\
\hline MEWS1 & 69.6 & MEWS2 & 64 & 0.333 \\
\hline MEWS1 & 69.6 & MEWS3 & 65.79 & 0.07 \\
\hline MEWS1 & 69.6 & WPSS & 70.73 & 0.845 \\
\hline MEWS1 & 69.6 & PEWS & 69.88 & 0.069 \\
\hline MEWS2 & 64 & MEWS3 & 65.79 & 0.845 \\
\hline MEWS2 & 64 & WPSS & 70.73 & 0.182 \\
\hline MEWS2 & 64 & PEWS & 69.88 & 0.319 \\
\hline MEWS3 & 65.79 & WPSS & 70.73 & 0.179 \\
\hline MEWS3 & 65.79 & PEWS & 69.88 & 0.069 \\
\hline WPSS & 70.73 & PEWS & 69.88 & 0.859 \\
\hline
\end{tabular}

2c: AUCs were tested for significant differences. Significance was assumed for $p<0.05$, marked by colored background. MEWS1, WPSS and PEWS were significantly better at predicting mortality in comparison to HbAlc. Abbreviations: AUC, Area under the curve; CART, Cardiac Arrest Risk Triage; MEWS, Modified Early Warning System; MTS, Manchester Triage System; PEWS, Patientrack Early Warning System; WPSS, Worthington Physiological Scoring System.
TA B L E 3. Distribution of Endpoints.

$\begin{array}{lc}\text { Admission to ICU, No. , \% } & 213(19.1 \%) \\ \text { LOS, median (IQR), days } & 6(3,10) \\ \text { LOS, mean (standard deviation), days } & 8.2(7.9) \\ \text { Death, No. , \% } & 35(3.1) \\ \text { Transferral, No. , \% } & 49(4.4 \%)\end{array}$

Abbreviations: LOS, legth of stay; IQR, interquartile range.

parameters absolute and relative frequencies were calculated.

Mean values for LOS of different subgroups were compared using Student's t-test.

Percentages for mortality and ICU transferral were compared using the Mann-Whitney-test.

Reciever-Operator-Characteristic (ROC) curves were calculated, graphically showing the predictive power for each test regarding the endpoints. Sensitivity is plotted vertically, whilst 1-specificity (i.e. the false positive rate) is plotted horizontally. The AUC of two ROC curves were compared using Delong's test [18]. The resulting p-values of the Delong's test were adjusted using the method of Benjamini and Hochberg [19].

\section{Results}

During the observation period, 1202 medical patients were admitted to the ED. 85 patients were excluded from further analyses because of missing data, resulting in a final study group of 1117 patients (Table 2) which were observed until the endpoints (Table 3).

In addition to the quartiles, cut offs were chosen at $\mathrm{HbA} 1 \mathrm{c}$ levels of $6.5 \%$ and $5.7 \%$. Values above $6.5 \%$ indicating a poorly controlled or undiagnosed diabetes mellitus, $5.7 \%$ to $6.5 \%$, indicating a pre-diabetic metabolic state while values below $5.7 \%$ indicate either a well-controlled diabetes or no diabetes at all.

The mean LOS for the combined lower two quartiles of HbAlc was 7.5 days while for the two higher quartiles it was 8.7 days (Table 4 ). This difference was statistically significant $(\mathrm{p}=0.012)$. Mean LOS for quartile 3 (7.9 days) was significantly shorter than for quartile 4 (9.5 days, $\mathrm{p}=$ 0.010). The differences between the other quartiles did not reach significance (Fig. 1). 
TA B LE 4. Outcomes for HbA1c quartiles and relevant subgroups.

\begin{tabular}{|c|c|c|c|c|}
\hline HbA1c-level & ICU transferral, \% & LOS, median, d & LOS, mean, d & Mortality, \% \\
\hline First quartile $(\leq 5.4 \%)$ & 16.6 & 6 & 7.4 & 2.8 \\
\hline Second quartile $(>5.4 \%$ to $<5.8 \%)$ & 19.7 & 6 & 7.7 & 3.5 \\
\hline Third quartile $(5.8 \%$ to $<6.3 \%)$ & 20.7 & 7 & 7.97 & 3.3 \\
\hline Fourthquartile $(\geq 6.3 \%)$ & 19.4 & 7 & $9.5]^{x}$ & 3 \\
\hline$<6.5 \%$ & 18.6 & 6 & 7.8 & 3.2 \\
\hline$\geq 6.5 \%$ & 20.6 & 7 & $9.5]^{\star}$ & \multirow{3}{*}{$\begin{array}{l}3.2 \\
3.1\end{array}$} \\
\hline $5.7 \%-\leq 6.5 \%$ & 19.3 & 6 & 8.2 & \\
\hline$<5.7 \%$ & 17.9 & 6 & 7.4 & \\
\hline
\end{tabular}

Length of stay was prolonged in subgroups with high HbAlc levels. Patients with levels above the median (quartiles $3+4)$ stayed significantly longer than those with levels below (quartiles 1+2). Among those with high levels, the fourth quartile stayed significantly longer than the third. The LOS for subgroups divided by HbAlc levels of $6.5 \%$ and $5.7 \%$ also differed significantly. The rate of ICU transfers showed a trend that did not reach significance. Mortality was poorly predicted by HbAlc. Abbreviations: d, days; ICU, Intensive/intermediate care unit; LOS, Length of stay; *, $p<0.05$.

Mean LOS for patients with a HbA1c below $6.5 \%$ was significantly shorter than for those with higher levels $(7.8$ days vs. 9.5 days, $p=0.004)$.

Mean LOS for patients with a HbA1c between $5.7 \%$ and $6.5 \%$ was not significantly different from those with lower levels $(p=0.143)$ but significantly shorter than those with values above $6.5 \%(\mathrm{p}=0.036)$.

While showing a trend for better outcomes for lower $\mathrm{HbA1c}$ values, across all subgroups neither the differences in mortality ( $\mathrm{p}=0.413$ to 0.971$)$ nor in ICU transferral $(\mathrm{p}=$ 0.193 to 0.942 ) were significant (Fig. 2).

Notably, $\mathrm{HbA} 1 \mathrm{c}$ values hardly correlated with mortality with an AUC of 0.501.

In predicting LOS, HbAlc (AUC $=0.568)$ provided similar results in comparison with the physiological scoring systems. The differences were not statistically significant $(\mathrm{p}=0.688$ to 0.714$)$.

All tested physiological scores showed some predictive ability regarding LOS with WPSS (AUC $=0.594)$ being significantly superior to the other MEWS variants (MEWS1: $\mathrm{AUC}=0.546, \mathrm{p}=0.009 ;$ MEWS2: $\mathrm{AUC}=0.550, \mathrm{p}=0.035$; MEWS3: $\mathrm{AUC}=0.544, \mathrm{p}=0.009$; PEWS: $\mathrm{AUC}=0.547$, $\mathrm{p}=0.009)$ and CART $(\mathrm{AUC}=0.588)$ reaching significance in comparison to MEWS1 $(p=0.043)$, MEWS3 $(p=0.035)$ and PEWS $(p=0.018)$. The predictive power of the Manchester Triage System was on the lower end with an AUC of 0.547 but not significantly different $(\mathrm{p}=0.180$ to 0.984).

For prediction of ICU transferral, HbAlc showed a positive correlation with an AUC of 0.525. This was not significantly different from the physiological systems $(p=$ 0.077 to 0.830 ).

Here, the highest AUC was reached by MTS (AUC = $0.636)$, reaching significance in comparison with $\mathrm{HbAlc}(\mathrm{p}$ $=0.001)$, CART $(p=0.003)$ and MEWS2 $(p<0.001)$.

In regard to predicting ICU transfer, among the physiological scoring systems WPSS (AUC $=0.587$ ), PEWS $($ AUC $=0.589)$, MEWS1 $(\mathrm{AUC}=0.587)$, and MEWS3
$(A U C=0.583)$ performed significantly better than MEWS2 (AUC $=0.501, p<0.001$ for WPSS, $\mathrm{p}=0.001$ for PEWS, $\mathrm{p}$ $=0.001$ for MEWS 1 and $p=0.001$ for MEWS3), and CART (AUC $=0.534, p=0.029$ for WPSS, $p=0.018$ for PEWS, $\mathrm{p}=0.021$ for MEWS1 and $\mathrm{p}=0.035$ for MEWS3).

\section{Discussion}

In this large prospective study, we examined a number of methods of triage and evaluation of severity of illness in emergency patients. We compared HbAlc levels as a potential new parameter to the MTS and several scoring systems based on physiological parameters.

We found that HbA1c correlated with LOS and need for intensive care in unselected medical emergency patients. This is in accordance with recent studies which linked glycated hemoglobin to adverse outcomes in cardiovascular [4] and neurovascular [5] events.

The predictive power of $\mathrm{HbAlc}$-levels regarding LOS was on par with the other tested methods, outperforming five of the seven competitors, underlining its potential as a possible tool in the ED.

The results regarding ICU transferral showed a positive correlation with $\mathrm{HbAlc}$-levels and their predictive power was not significantly different from the six tested physiological scoring systems. Only the MTS performed significantly better here.

None of the tested methods was clearly superior in predicting all of our three endpoints. Among the (M)EWS variants, WPSS was the most useful tool in predicting negative outcomes. The MTS provided mixed results.

Considering the complexity of the physiological scoring systems which are calculated using weighted conversions of four to six parameters, we deem these results as noteworthy for a single parameter.

Interestingly, the correlation between elevated $\mathrm{HbAlc}$ and mortality was rather poor $(\mathrm{AUC}=0.501)$. With an overall mortality of only $3.1 \%$ relatively few patients fell 
into this group. A group of patients overrepresented in this group had oncological diagnoses, often suffering from advanced disease. These patients tend to receive continous medical care and are as such unlikely to suffer from uncontrolled or undetected diabetes. This group of patients suffered high mortality $(40.0 \%$ of deceased versus $13.0 \%$ of the overall cohort, data not shown), which may partly explain our results regarding the correlation of mortality and $\mathrm{HbA} 1 \mathrm{c}-\mathrm{levels}$.

The relatively high prevalence of oncological diagnoses in our cohort may be a limiting factor with respect to the generalisability of our findings.

\section{Conclusions}

Our study showed that HbA1c levels correlate with clinical outcomes of emergency patients, largely comparable with established methods of triage like MTS and (M)EWS variants.

Determination of HbA1c may provide useful additional information to identify patients at risk and may be a candidate for inclusion into early warning systems in the form of point-of-care testing.

Our data suggests that this may prove helpful in improving accuracy. In our opinion, this should be the target of further study.

\section{ACKNOWLEDGMENTS}

We would like to thank Dr. Friedrich Pahlke for advice on statistical analysis.

\section{CONFLICTS OF INTEREST}

The authors declare that there is no conflict of interest regarding the publication of this article.

\section{DATA AVAILABILITY}

The data used to support the findings of this study are available from the corresponding author upon request.

\section{FUNDING INFORMATION}

This research received no specific grant from any funding agency in the public, commercial, or not-for-profit sectors. At the time of data acquisition, the authors were employed at RoMed Clinical Centre Rosenheim, Pettenkoferstr. 10, 83022 Rosenheim, Germany, where the study was conducted.

\section{REFERENCES}

[1] Brabrand M, Folkestad L, Groes Clausen N, Knudsen T, Hallas J. Risk scoring systems for adults admitted to the emergency department: a systematic review. Scand J Trauma Resus. 2010;18:8
[2] McNarry AF, Goldhill DR. Simple bedside assessment of level of consciousness: comparison of two simple assessment scales with the Glasgow Coma scale. Anaesthsia. 2004;59(1):34-37

[3] Manchester Triage Group. Mackway-Jones K, ed. Emergency triage. London: BMJ Publishing Group, 1997.

[4] Churpek MM, Yuen TC, Park SY, Meltzer DO, Hall B , Edelson DP. Derivation of a cardiac arrest prediction model using ward vital signs. Crit Care Med. 2012;40:2102-2108.

[5] Churpek MM, Yuen TC, Huber MT, Park SY, Hall JB, Edelson DP. Predicting cardiac arrest on the wards: a nested case-control study. Chest. 2012;141:1170-6.

[6] Recine U, Scotti E, Bruzzese V, D’Amore F, Manfelotto D, Simonelli I, et al. The Change of Hospital Internal Medicine: A Study on Patients admitted in Internal Medicine Wards of 8 Hospitals of the Lazio Area, Italy. Ital J Med. 2015;9:252-259

[7] Subbe CP, Kruger M, Rutherford P, Gemmel L. Validation of a modified Early Warning Score in medical admissions. Q J Med. 2001;94:521-526

[8] Duckitt RW, Buxton-Thomas R, Walker J, Cheek E, Bewick V, Venn $\mathrm{R}$, et al. Worthing physiological scoring system: derivation and validation of a physiological early-warning system for medical admissions. An observational, population-based single-centre study. Br J Anaesth. 2007;98:769-74.

[9] Jones S, Mullally M, Ingleby S., Buist M, Bailey M, Eddleston J $\mathrm{M}$. Bedside electronic capture of clinical observations and automated clinical alerts to improve compliance with an Early warning Score protocol. Crit Care Resus. 2011;13:83-88

[10] Chen CL, Yen DH, Lin CS, Tsai SH, Chen SJ, Sheu WH, et al. Glycated hemoglobin level is an independent predictor of major adverse cardiac events after nonfatal acute myocardial infarction in nondiabetic patients. Medicine (Baltimore). 2017;96:e6743.

[11] Gornik I, Gornik O, Gasparovic V. HbA1c is outcome predictor in diabetic patients with sepsis. Diabetes ResClinPract.2007;77:120 125.

[12] Lorea E, Morhain H, García-Agulló O, de Esteban M, Beroiz I, Culleré G. Prognostic value of blood glucose in emergency room and glycosylated hemoglobin in patients who have suffered an acute cerebro-vascular event. Med Clin (Barc). 2017;S0025-7753:30032-5.

[13] Liu Y, Yang YM, Zhu J, Tan HQ, Liang Y, Li JD. Prognostic significance of hemoglobin A1c level in patients hospitalized with coronary artery disease. A systematic review and meta-analysis. Cardiovasc Diabetol. 2011;10: 98.

[14] Magee MF, Nassar C. Hemoglobin A1c Testing in an Emergency Department. J Diabetes Sci Technol. 2011;5: 1437-1443.

[15] Beagley J, Guariguata L, Weil L, Motala AA. Global estimates of undiagnosed diabetes in adults. Diabetes Res Clin Pract. 2014;103:150 160

[16] Global report on diabetes. Geneva: World Health Organization; 2016

[17] Robin X, Turck N, Hainard A, Tiberti N, Lisacek F, Sanchez JC et al. pROC: an open-source package for $\mathrm{R}$ and $\mathrm{S}+$ to analyze and compare ROC curves. BMC Bioinformatics. 2011;12:77.

[18] DeLong ER, DeLong DM, Clarke-Pearson DL. Comparing the areas under two or more correlated receiver operating characteristic curves: a nonparametric approach. Biometrics. 1988;44:837-845.

[19] Benjamini Y, Hochberg Y. Controlling the false discovery rate: a practical and powerful approach to multiple testing. J R Stat Soc B. 1995;57:289-300.

How to cite this article: Markus Sander, Johannes Fickler, Uwe Neddermeyer, et al. Predictive Value of HbA1c-levels with Regard to In-hospital Mortality, Length of Hospital Stay and Intensive Care Utilisation versus Different Emergency Risk Scores and the Manchester Triage System in Unselected Medical Emergency Admissions. Signa Vitae. 2020;16(1):39-45. doi:10.22514/sv.2020.16.0006. 